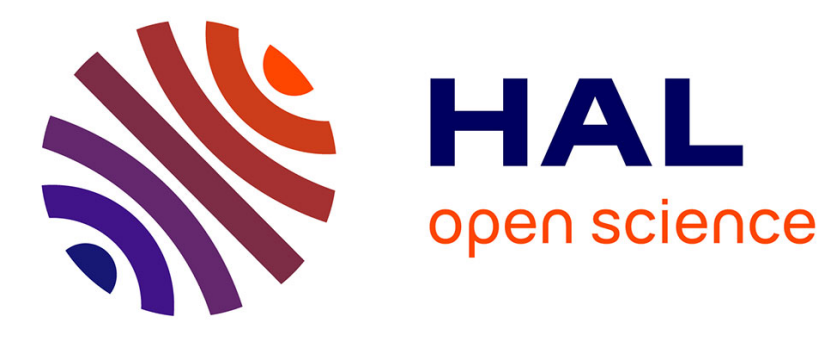

\title{
On the decentralized management of scrambling codes in small cell networks
}

\author{
Samir M. Perlaza, Zhu Han, H. Vincent Poor, Dusit Niyato
}

\section{To cite this version:}

Samir M. Perlaza, Zhu Han, H. Vincent Poor, Dusit Niyato. On the decentralized management of scrambling codes in small cell networks. 13th IEEE International Conference on Communication Systems (ICCS), Nov 2012, Singapour, Singapore. pp.478-482, 10.1109/ICCS.2012.6406194 . hal01281184

\section{HAL Id: hal-01281184 \\ https://hal.science/hal-01281184}

Submitted on 1 Mar 2016

HAL is a multi-disciplinary open access archive for the deposit and dissemination of scientific research documents, whether they are published or not. The documents may come from teaching and research institutions in France or abroad, or from public or private research centers.
L'archive ouverte pluridisciplinaire HAL, est destinée au dépôt et à la diffusion de documents scientifiques de niveau recherche, publiés ou non, émanant des établissements d'enseignement et de recherche français ou étrangers, des laboratoires publics ou privés. 


\title{
On the Decentralized Management of Scrambling Codes in Small Cell Networks
}

\author{
Samir M. Perlaza ${ }^{\dagger}$, Zhu Han ${ }^{\ddagger}$, H. Vincent Poor ${ }^{\dagger}$ and Dusit Niyato ${ }^{\star}$. \\ ${ }^{\dagger}$ Dept. EE, Equad 19 Olden Street, Princeton University, Princeton, NJ 08544, USA \\ ${ }^{\star}$ Dept. CE, Block N4-02a-32, Nanyang Avenue, Nanyang Technological University, 639798, Singapore \\ ${ }^{\mp}$ Dept. ECE, N324, Engineering Building 1, University of Houston, Houston, TX 77004, USA. \\ \{perlaza, poor\}@princeton.edu, dniyato@ntu.edu.sg, zhan2@mail.uh.edu.
}

\begin{abstract}
In this paper, the problem of primary scrambling code (PSC) allocation in wideband code-division multiple access (WCDMA) self-configuring small cell networks is studied using tools from game theory. In this game, it is shown that when the number of available scrambling codes is larger than or equal to the number of small cells, globally optimal and individually optimal PSC allocations always exist and coincide with the set of Nash equilibria. In the converse case, it is shown that an individually optimal PSC allocation might not exist. However, the existence of a global optimal allocation is always ensured. Here, the notion of individual optimality corresponds to the case in which small cells cannot reduce their probabilities of code confusion by changing their own PSC choices. On the other hand, the notion of global optimality refers to the case in which no other PSC allocation can reduce the probability of code confusion. The second contribution is a pair of algorithms based on the notion of trial and error learning that allow the achievability of an individually optimal PSC allocation, if it exists, or a globally optimal allocation otherwise. Both algorithms present attractive properties independently of the network topology and the ratio between the numbers of PSCs and active small cells in the network.
\end{abstract}

\section{INTRODUCTION}

The problem of primary scrambling code (PSC) selection has become one of the most prominent problems in wideband code-division multiple access (WCDMA) cellular networks due to the evolution of these systems towards partially or fully decentralized networks [1]. Typically, in cellular systems such as UMTS and HSPA, PSCs are used as the main cell identifiers and thus, neighboring cells must use different PSCs in order to facilitate cell association and handovers [2]. In current systems, the allocation of PSCs is done manually [3] or using centralized algorithms based on the ideas of cluster reuse techniques [4], [5], graph coloring [6] or similar algorithms. As long as the PSC management is performed in a centralized fashion, there always exists an optimal allocation, that is, an allocation that minimizes the PSC confusion in the network. However, the inclusion of self-configuring small-cell systems, often deployed in an ad hoc and unplanned manner, constrains the centralized approach and requires the PSC management to be done in a decentralized fashion.

Decentralized PSC management is particularly troublesome for the following reasons. First, as small cells might be

This research was supported in part by the Army Research Office under MURI Grant $W 911 N F-11-1-0036$, the Qatar National Research Fund (QNRF) and the U.S. National Science Foundation Grants CNS-1117560, ECCS-1028782, CNS-0953377 deployed in the same coverage area of legacy macrocells, a subset of all available PSCs must be reserved for the use of small cells. Moreover, this set of PSCs must be included in the neighbor cell list (NCL) of all legacy macrocells. In order to avoid long NCLs and thus reduce the neighbor detection and cell association time, often the set of PSC reserved for small cells is small. This implies that in high density networks, there often exist more small cells than available PSCs. Second, as small cells are deployed dynamically without following any network planning, PSC management must be dynamic which can significantly increase the demand of network signaling among small-cells. Hence, PSC management must be both dynamic and low/zero demanding in terms of message passing requirements among small cells.

The problem of decentralized PSC management has been formalized for the first time in [7] as a decentralized optimization problem. Therein, several mechanisms for decentralized PSC allocation are proposed. However, as acknowledged by the authors in [7], often those mechanisms fail to achieve a scrambling code (SC) allocation that minimizes the code confusion as the number of small base stations increases. In this paper, we use tools from game theory to re-formulate the decentralized PSC allocation problem as a game in a strategic form and we introduce the notions of individually and globally optimal PSC allocations. An individually optimal allocation refers to the case in which players cannot reduce their probability of code confusion by changing their own PSC choices. This recasts the notion of Nash equilibrium in the game. We refer to [8] for a comprehensive discussion on the relevance of the notion of equilibrium. A globally optimal PSC allocation refers to the case where no other PSC allocation can reduce the probability of code confusion, which recalls the notion of welfare maximization. The existence of at least one globally optimal PSC allocation is always ensured. Moreover, we show that in low-density networks, where there are at least as many PSCs as small cells, any individually optimal PSC allocation is also globally optimal and vice versa. Conversely, when there are more small cells than PSCs, the existence of an individually optimal PSC allocation is not ensured, and if it exists, it is not necessarily globally optimal.

The main difference between individual and global optimality of PSC allocations often can be described in terms of fairness. For instance, to achieve a globally optimal PSC allocation, some small cells must forego maximization of their individual probabilities of code confusion. That is, an 
individually optimal PSC allocation can be seen as the most fair allocation, while a globally optimal allocation can be interpreted as the most efficient allocation. Given the relevance of these two types of PSC allocations, we use the notions of trial and error learning [9]-[11] to provide fully decentralized algorithms that achieve either an individually optimal PSC allocation, if it exists, or a globally optimal PSC allocation.

\section{Problem Formulation}

Consider a set $\mathcal{K}=\{1, \ldots, K\}$ of small base stations aiming to autonomously choose their SCs. There exists a finite set of $A$ SCs that must be reused throughout the network. We associate to each SC one of the elements of the set $\mathcal{A}=\{1, \ldots, A\}$ for its identification. For ease of presentation, we assume that small base stations use unique scrambling codes which corresponds to the case in which small cells do not implement any cell sectorization. We denote by $a_{k} \in \mathcal{A}$ the index of the scrambling code chosen by small base station $k$. A full code allocation is denoted by the vector $\boldsymbol{a}=\left(a_{1}, \ldots, a_{K}\right) \in \mathcal{A}^{K}$. Small base station $k$ gathers observations $r_{k}\left(\ell_{j}\right)$ of the receiver signal code power (RSCP) reported by device $j$ located in coordinates $\ell_{j} \in \mathbb{R}^{+}$via the corresponding common pilot channel $(\mathrm{CPICH})$. This measure is used to determine the strength of the signal of small base station $k$ at location $\ell_{j}$. Small-base station $k$ calculates the ratios $m_{k j} \in \mathbb{R}^{+}$, for all $j \in \mathcal{K}$, where

$$
m_{k j}=\max _{\ell \in \mathcal{L}_{k}} \frac{r_{k}(\ell)}{r_{j}(\ell)},
$$

and $\mathcal{L}_{k}$ is the set of locations where small base station $k$ was able to observe the RSCP. Note that when $m_{k j} \rightarrow 0$, base station $k$ must avoid using the same $\mathrm{SC}$ as base station $j$. On the contrary, when $m_{k j} \rightarrow \infty$, base station $k$ becomes more tolerant to using the same SC used by base station $j$. This is basically because, a large $m_{k j}$ might refers to a sufficiently large distance from cell $k$ to cell $j$ and thus it yields a low probability of code confusion. Note also that, a ratio $m_{j k}$ might differ from $m_{k j}$ depending on the network configuration. Often, this parameter depends on the propagation conditions, antenna design and physical implementation parameters such as the height and tilt of the antennas of each base station.

The aim of small cell $k$ is to autonomously choose its SC according to $m_{k 1}, \ldots, m_{k K}$ in order to minimize the coding confusion in the network.

\section{GAME FORMULATION}

We model the problem of SC selection in small cell networks using tools from game theory. Consider a game in normal form,

$$
\mathcal{G}=\left(\mathcal{K},\{\mathcal{A}\}_{k \in \mathcal{K}},\left\{u_{k}\right\}_{k \in \mathcal{K}}\right),
$$

and let the sets $\mathcal{K}$ and $\mathcal{A}$ be the set of small base stations and the set of SCs, respectively. That is, in the game $\mathcal{G}$, players are the small base stations and the actions of each player are the choices among the available SCs. In the following, we indifferently refer to the small base stations as players and vice versa. An outcome of the game is indeed a full set of SCs $a \in \mathcal{A}^{K}$ to which we refer as an action profile. Often, with a slight abuse of notation, for a given proper subset $\mathcal{S}$ of $\mathcal{K}$, with $|\mathcal{S}|=S$, we write $\boldsymbol{a}=\left(\boldsymbol{a}_{\mathcal{S}}, \boldsymbol{a}_{-\mathcal{S}}\right)$, where $\boldsymbol{a}_{\mathcal{S}}=\left(a_{n_{1}}, \ldots, a_{n_{S}}\right)$ and $n_{s} \in \mathcal{S}$ for all $s \in\{1, \ldots, S\}$. Similarly, $\boldsymbol{a}_{-\mathcal{S}}=\left(a_{n_{1}}, \ldots, a_{n_{K-S}}\right)$ with $n_{s} \in \mathcal{K} \backslash \mathcal{S}$ for all $s \in\{1, \ldots, A-S\}$. When, $\mathcal{S}$ is a singleton, for instance $\mathcal{S}=\{k\}$, we write $\boldsymbol{a}=\left(a_{k}, \boldsymbol{a}_{-k}\right)$.

The interest of each player is to maximize its utility function $u_{k}: \mathcal{A}^{K} \rightarrow \mathbb{R}^{+}$defined as follows:

$$
u_{k}\left(a_{k}, \boldsymbol{a}_{-k}\right)=\sum_{j=1}^{K} w_{k j} \mathbb{1}_{\left\{a_{k} \neq a_{j}\right\}},
$$

with $w_{k j}=\frac{1}{m_{k j}}>0$. Note that $u_{k}\left(a_{k}, \boldsymbol{a}_{-k}\right) \rightarrow 0$ implies that all small base station are using the same SC. Conversely, $u_{k}\left(a_{k}, \boldsymbol{a}_{-k}\right) \rightarrow u_{k, \max }$, with $u_{k, \max }=\sum_{j=1}^{K} w_{k j}$, the maximum achievable utility, implies that small base stations use different SCs from each other. A full SC allocation $\boldsymbol{a}^{*}$ is an optimal allocation from the point of view of small base station $k$ if the utility $u_{k}\left(a_{k}^{*}, \boldsymbol{a}_{k}^{*}\right)$ is maximized by the SC $a_{k}$ given the choices of all the other small base stations $\boldsymbol{a}_{-k}^{*}$. This game outcome corresponds to the notion of Nash equilibrium (NE), which we define as follows,

Definition 1 (Nash Equilibrium in Pure Strategies): An action profile $\boldsymbol{a}^{*}$ is an $N E$ if, for all players $k \in \mathcal{K}$ and $\forall a_{k}^{\prime} \in \mathcal{A}$,

$$
u_{k}\left(a_{k}^{*}, \boldsymbol{a}_{-k}^{*}\right) \geqslant u_{k}\left(a_{k}^{\prime}, \boldsymbol{a}_{-k}^{*}\right) .
$$

\section{A. Existence of the NE}

The existence of an equilibrium in the game $\mathcal{G}$ is strongly related to the number of small base stations and available SCs. For instance, in the case in which there are at least the same number of SCs as small base stations $(A \geqslant K)$, then several equilibria might exist. Indeed, a full code allocation $\boldsymbol{a}$, with $a_{j} \neq a_{i}$, for all $(i, j) \in \mathcal{K}^{2}$ and $i \neq j$ is an NE. In the converse case $(A<K)$, which might be the case for dense networks, an NE simply might not exist. We provide an example to illustrate this case.

Example 1: Consider a network with $K=3$ small base stations and $A=2$ scrambling codes. The achieved utilities are described in the following tables. Note that if the

\begin{tabular}{|c|c|c|}
\hline$P_{1} \backslash P_{2}$ & $a_{2}=1$ & $a_{2}=2$ \\
\hline$a_{1}=1$ & $(0,0,0)$ & $\left(w_{12}, w_{21}+w_{23}, w_{32}\right)$ \\
\hline$a_{1}=2$ & $\left(w_{12}+w_{13}, w_{21}, w_{31}\right)$ & $\left(w_{13}, w_{23}, w_{31}+w_{32}\right)$ \\
\hline
\end{tabular}

Fig. 1. Utilities achieved in the game $\mathcal{G}=\left(\mathcal{K},\{\mathcal{A}\}_{k \in \mathcal{K}},\left\{u_{k}\right\}_{k \in \mathcal{K}}\right)$ with action profiles of the form $\left(a_{1}, a_{2}, 1\right)$. Here for any tuple $\left(x_{1}, x_{2}, x_{3}\right), x_{i}=$ $u_{i}\left(a_{1}, a_{2}, 1\right)$, for all $i \in\{1,2,3\}$. Player 1 choose rows and player 2 chooses columns.

coefficients of the utility functions simultaneously satisfy, at least, the following conditions: $w_{12}<w_{13}, w_{23}<w_{21}$ and $w_{31}<w_{32}$, then an NE does not exists.

The reasoning above leads us to the following result.

Lemma 1 (Existence of the NE): $\quad$ The game $\mathcal{G}=\left(\mathcal{K},\{\mathcal{A}\}_{k \in \mathcal{K}},\left\{u_{k}\right\}_{k \in \mathcal{K}}\right)$ has at least one $N E$ when $A \geqslant K$. Conversely, when $A<K$, the existence of one $N E$ is not ensured. 


\begin{tabular}{|c|c|c|}
\hline$P_{1} \backslash P_{2}$ & $a_{2}=1$ & $a_{2}=2$ \\
\hline$a_{1}=1$ & $\left(w_{13}, w_{23}, w_{31}+w_{32}\right)$ & $\left(w_{12}+w_{13}, w_{21}, w_{31}\right)$ \\
\hline$a_{1}=2$ & $\left(w_{12}, w_{21}+w_{23}, w_{32}\right)$ & $(0,0,0)$ \\
\hline
\end{tabular}

Fig. 2. Utilities achieved in the game $\mathcal{G}=\left(\mathcal{K},\{\mathcal{A}\}_{k \in \mathcal{K}},\left\{u_{k}\right\}_{k \in \mathcal{K}}\right)$ with action profiles of the form $\left(a_{1}, a_{2}, 2\right)$. Here for any tuple $\left(x_{1}, x_{2}, x_{3}\right), x_{i}=$ $u_{i}\left(a_{1}, a_{2}, 2\right)$, for all $i \in\{1,2,3\}$. Player 1 choose rows and player 2 chooses columns.

\section{B. Multiplicity of the $N E$}

In Example 1, we have shown that, depending on the coefficients of the utility functions $w_{i j}$, an NE might or might not exist when $K>A$. However, when $K \leqslant A$, the existence of at least one equilibria is guaranteed. That is, an action profile $\boldsymbol{a}^{*}$ such that for all $(i, j) \in \mathcal{K}^{2}$ with $i \neq j, a_{j}^{*} \neq a_{i}^{*}$ always exists. This corresponds to the case in which each small base station uses a different SC. In this particular case, if $\boldsymbol{a}^{*}$ is an $\mathrm{NE}$, then for all $k \in \mathcal{K}$,

$$
u_{k}\left(a_{k}^{*}, \boldsymbol{a}_{-k}^{*}\right)=\sum_{i=1}^{K} w_{k i}=u_{k, \max } .
$$

Note that if player $k$ unilaterally deviates from $\boldsymbol{a}^{*}$ and uses another SC such that $a_{k}=a_{i}^{*}$, with $i \in \mathcal{K} \backslash\{k\}$, it follows that

$$
u_{k}\left(a_{k}^{*}, \boldsymbol{a}_{-k}^{*}\right)-u_{k}\left(a_{k}, \boldsymbol{a}_{-k}^{*}\right)>w_{k i}
$$

and thus, under the assumption that $w_{i j}>0$ for all $(i, j) \in \mathcal{K}$, an SC allocation $\boldsymbol{a}^{*}$ is an equilibrium if and only if every small base station uses a different SC. Interestingly, in this case, all the NEs achieve the same utility (6), which is the maximum achievable utility. This reasoning leads to the following result.

Lemma 2 (Multiplicity of the NE): Let $\mathcal{A}_{\mathrm{NE}} \subseteq \mathcal{A}^{K}$ be the set of $N E$ of the game $\mathcal{G}=\left(\mathcal{K},\{\mathcal{A}\}_{k \in \mathcal{K}},\left\{u_{k}\right\}_{k \in \mathcal{K}}\right)$, with $A \geqslant K$, and assume that for all $(i, j) \in \mathcal{K}^{2}, w_{i j}>0$. Then,

$$
\left|\mathcal{A}_{\mathrm{NE}}\right|=\frac{A !}{(A-K+1) !},
$$

and for all $k \in \mathcal{K}, u_{k}\left(a_{k}^{*}, \boldsymbol{a}_{-k}^{*}\right)=u_{k, \max }$.

In the case in which $K>A$, it is also possible to observe the existence of several equilibria, as we shall see later in Example 3. Indeed, an interesting point in Example 3 is that even if several NE exist, they can lead to the same individual utilities. This implies that no preference for a particular NE can be established. However, for a large number of small base stations and SCs, the number of equilibria can be significantly large and often, at each NE, small base stations obtain different utilities. We shall illustrate this observation in Example 2 in the next section. In this context, the natural question is then how to select a particular NE. In the following section, we analyze thoroughly this issue.

\section{Optimal Scrambling Code Selection}

\section{A. Global and Individual Optimality}

A scrambling code allocation is individually optimal if it is an NE. That is, given the SCs adopted by all small base stations no small base station can improve its own utility by selecting another SC. To analyze the global optimality of a given SC allocation, we define a function $g: \mathcal{A}^{K} \rightarrow \mathbb{R}$ to which we refer as the welfare function. We define $g$ as follows:

$$
g(\boldsymbol{a})=\sum_{i=1}^{K} u_{i}(\boldsymbol{a}) \leqslant \sum_{i=1}^{K} u_{i, \max }
$$

We use the welfare function to define the optimality of an $\mathrm{SC}$ allocation.

Definition 2 (Optimal SC Allocations): An SC allocation $\boldsymbol{a}^{*}$ is said to be globally optimal if it maximizes the welfare function. That is, for all $\boldsymbol{a}^{\prime} \in \mathcal{A}^{K}, g\left(\boldsymbol{a}^{\prime}\right) \leqslant g\left(\boldsymbol{a}^{*}\right)$.

Note that when an SC allocation $\boldsymbol{a}$ is globally optimal, it assigns different SCs to the pairs of base stations $(i, j)$ for which the parameters $w_{i j}$ or $w_{j i}$ are among the highest in the network. That is, the pairs of small base stations $(i, j)$ whose ratios of RSCP measures $m_{j i}$ or $m_{i j}$ are among the weakest in the network use different SCs. This clearly minimizes the probability of code confusion in the network.

From Lemma 1, under the assumption that $A \geqslant K$, it becomes clear that any NE of the game $\mathcal{G}$ is a globally optimal SC allocation. Moreover, any globally optimal SC allocation is an NE. In the converse case $(K>A)$, an SC allocation that is an NE might not necessarily be globally optimal. Moreover, a globally optimal SC allocation is not necessarily an NE. We provide the following example to elucidate this observation.

Example 2: Consider a network with $K=4$ small base stations, $A=3$ scrambling codes and the following network parameters: $w_{12}=w_{21}=w_{13}=w_{31}=w_{24}=w_{42}=$ $w_{34}=w_{43}=w, w_{14}=w_{41}=w_{1}$ and $w_{23}=w_{32}=$ $w_{2}$. Then, under the conditions that $w_{1}<w_{2}<w$, the SC allocations $\boldsymbol{a}^{(1)}=(1,2,2,3)$ and $\boldsymbol{a}^{(2)}=(1,2,3,1)$ are both NEs. However, $g\left(\boldsymbol{a}^{(1)}\right)<g\left(\boldsymbol{a}^{(2)}\right)$ which implies that $\boldsymbol{a}^{(1)}$ is an NE but it is not globally optimal. On the contrary, it can be verified that $\boldsymbol{a}^{(2)}$ is both an NE and a globally optimal SC allocation.

Example 3: Consider a network with $K=3$ small base stations and $A=2$ scrambling codes. The achieved utilities are presented in Fig. 1 and 2. Note that if the coefficients of the utility functions simultaneously satisfy, at least, the following conditions, $w_{12}<w_{13}, w_{21}<w_{23}, w_{31}<w_{32}$ and $w_{23}+$ $w_{32}<w_{12}+w_{21}$, then the action profiles $\boldsymbol{a}^{(1)}=(1,1,2)$ and $\boldsymbol{a}^{(1)}=(2,2,1)$ are both NEs and for all $i \in \mathcal{K}, u_{i}\left(\boldsymbol{a}^{(1)}\right)=$ $u_{i}\left(\boldsymbol{a}^{(2)}\right)$. However, these NEs are not globally optimal given that $g\left(\boldsymbol{a}^{(1)}\right)=g\left(\boldsymbol{a}^{(2)}\right)<g(1,2,2)$.

An interesting trade-off between global and individual optimality would be the type of SC allocations $a^{*}$ that satisfy the following condition:

$$
\boldsymbol{a}^{*} \in \arg \max _{\boldsymbol{a} \in \mathcal{A}_{\mathrm{NE}}} g(\boldsymbol{a}) .
$$

That is, individually optimal SC allocations that are the closest to a globally optimal SC allocation. We refer to the SC allocations that satisfy the condition in (8) as welfaremaximizing individually optimal SC allocations.

\section{Achieving Optimal SC Allocations}

In this section, we describe a set of mechanisms that allow small base stations to achieve welfare-maximizing individually optimal SC allocations and globally optimal SC allocations in 
a fully decentralized fashion [12]. Before we start, we clarify that time is divided into equal-length intervals indexed by $n \in$ $\mathbb{N}$. At interval $n$, small base station $k$ can update its SC choice $a_{k}(n)$ and it is able to calculate the value of its achieved utility $u_{k}\left(a_{k}(n), \boldsymbol{a}_{-k}(n)\right)$. A discussion of how to measure the RCSP and obtain $u_{k}\left(a_{k}(n), \boldsymbol{a}_{-k}(n)\right)$ is presented in [7].

\section{A. Achieving Welfare Maximizing Individually Optimal SC Allocations}

In Appendix B, we briefly describe the notion of learning by trial and error (TE) first introduced in [9], and lately refined in [10]. In the following, we refer to the TE algorithms presented in [10] as TE type I (TE-I). Our main result is presented in the following theorem.

Theorem 1: Assume all players of the game $\mathcal{G}=$ $\left(\mathcal{K},\{\mathcal{A}\}_{k \in \mathcal{K}},\left\{u_{k}\right\}_{k \in \mathcal{K}}\right)$ use trial and error learning (TE-I) with experimentation rate $\epsilon>0$. Then,

(i) if $\mathcal{G}$ has at least one $N E$, at every stochastically stable network state, the network uses a welfare maximizing and individually optimal SC allocation.

(ii) if $\mathcal{G}$ does not have an $N E$, at every stochastically stable network state, the network uses a globally optimal SC allocation.

In Theorem 1, the notion of stochastically stable state must be understood as a network configuration that is observed during a large proportion of the time the network is active. As we shall see in the next section, this proportion is often a function of the experimentation rate of the algorithm TE-I.

Finally, the proof of Theorem 1 follows from the fact that the game $\mathcal{G}$ is finite and interdependent (see Appendix A) and Theorem 1 in [10].

\section{B. Achieving Globally Optimal SC Allocations}

In Appendix $\mathrm{C}$, we briefly describe the refinement of the notion of learning by trial and error presented in [11] to which we refer as TE type II (TE-II). The main result regarding the achievability of globally optimal SC allocations is presented in the following theorem.

Theorem 2: Assume all players of the game $\mathcal{G}=$ $\left(\mathcal{K},\{\mathcal{A}\}_{k \in \mathcal{K}},\left\{u_{k}\right\}_{k \in \mathcal{K}}\right)$ use trial and error learning (TE-II) with $\epsilon>0$. Then, at every stochastically stable network state, the network uses a globally optimal SC allocation.

The proof of Theorem 2 follows from the fact that the game $\mathcal{G}$ is finite and interdependent (see Appendix A) and Theorem 1 in [11].

\section{Vi. CONCLUSIONS}

The problem of decentralized PSC allocation in WCDMA self-configuring small cell networks has been formulated as a strategic game. In the case of low-density networks, that is, when the number of available scrambling codes is bigger than the number of small cells, it has been shown that both globally optimal and individually optimal PSC allocations always exist and coincide with the set of Nash equilibria. In the case of high-density networks, it is shown that an individually optimal PSC allocation might not exist, however the existence of a globally optimal allocation is always ensured. Two algorithms based on the notion of trial and error learning have been presented as candidates for allowing the achievability of an individually optimal PSC allocation, if it exists, or a globally optimal allocation otherwise. Both algorithms present important properties such as ensured convergence independently of the topology of network and the number of PSCs or small cells in the network.

\section{REFERENCES}

[1] 3GPP, "Home Node B radio frequency (RF) requirements (FDD)," 3rd Generation Partnership Project (3GPP), TR 25.967 Version 10, Apr. 2011.

[2] S. Kourtis, "Code planning strategy for umts-fdd networks," in Proc. IEEE 51st Vehicular Technology Conference (VTC 2000 Spring), Tokyo, Japan, May 2000.

[3] T. Jansen, M. Amirijoo, U. Turke, L. Jorguseski, K. Zetterberg, R. Nascimento, L. Schmelz, J. Turk, and I. Balan, "Embedding multiple selforganisation functionalities in future radio access networks," in Proc. IEEE 69th Vehicular Technology Conference, (VTC Spring 2009), Barcelona, Spain, Apr. 2009.

[4] C. R. Chang, J. Wan, and M. Yee, "Pn offset planning strategies for nonuniform CDMA networks," in Proc. IEEE 47th Vehicular Technology Conference (VTC 1997 Spring), Phoenix, AZ, USA, May 1997.

[5] R. Joyce, T. Griparis, G. Conroy, B. Graves, and I. Osborne, "A novel code planning approach for a wcdma network," in Proc. 4th International Conference on $3 G$ Mobile Communication Technologies, London, United Kingdom, Jun. 2003.

[6] Y.-H. Jung and Y. Lee, "Scrambling code planning for 3gpp w-cdma systems," in Proc. IEEE 53rd Vehicular Technology Conference (VTC 2001 Spring), Rhodes, Greece, May 2001.

[7] A. Checco, R. Razavi, D. Leith, and H. Claussen, "Self-configuration of scrambling codes for wcdma small cell networks," in Proc. IEEE $23 r d$ International Symposium on Personal, Indoor and Mobile Radio Communications (PIMRC), Sydney, Australia, Sept. 2012.

[8] S. M. Perlaza, H. Tembine, S. Lasaulce, and M. Debbah, "Quality-ofservice provisioning in decentralized networks: A satisfaction equilibrium approach," IEEE Journal of Selected Topics in Signal Processing, vol. 6, no. 2, pp. 104-116, 2012.

[9] H. P. Young, "Learning by trial and error," Department of Economics, University of Oxford, Oxford, United Kingdom, Tech. Rep., Mar. 2009.

[10] B. S. Pradelski and H. P. Young, "Efficiency and equilibrium in trial and error learning," Department of Economics, University of Oxford, Oxford, United Kingdom, Tech. Rep., Jun. 2011.

[11] J. R. Marden, L. Y. Pao, and H. P. Young, "Achieving Pareto optimality through distributed learning," Department of Economics, University of Oxford, Oxford, United Kingdom, Tech. Rep., July. 2011.

[12] L. Rose, S. M. Perlaza, S. Lasaulce, and M. Debbah, "Learning equilibria with partial information in wireless networks," IEEE Communications Magazine, Special Issue Game Theory in Wireless Communications, vol. 49, no. 8, pp. 136-142, 2011.

\section{APPENDIX A}

\section{INTERDEPENDENCY OF THE GAME $\mathcal{G}$}

In this appendix, we show that under the assumptions that $w_{i j}>0$ for all $(i, j) \in \mathcal{K}^{2}$, the game $\mathcal{G}$ is interdependent. Roughly speaking, we show that there does not exist a bipartition of the set $\mathcal{K}$ such that players in one set do not interact with the players of the other set.

Assume that there exist two subsets $\mathcal{K}^{(1)} \subset \mathcal{K}$ and $\mathcal{K}^{(2)}=$ $\mathcal{K} \backslash \mathcal{K}^{(1)}$ such that there exists a pair of action profiles $\boldsymbol{a}^{*}=$ $\left(\boldsymbol{a}_{\mathcal{K}^{(1)}}^{*}, \boldsymbol{a}_{\mathcal{K}^{(2)}}^{*}\right)$ and $\boldsymbol{a}^{\prime}=\left(\boldsymbol{a}_{\mathcal{K}^{(1)}}^{\prime}, \boldsymbol{a}_{\mathcal{K}^{(2)}}^{*}\right)$ that satisfies for all $j \in \mathcal{K}^{(2)}$,

$$
u_{j}\left(\boldsymbol{a}_{\mathcal{K}^{(1)}}^{*}, \boldsymbol{a}_{\mathcal{K}^{(2)}}^{*}\right)=u_{j}\left(\boldsymbol{a}_{\mathcal{K}^{(1)}}^{\prime}, \boldsymbol{a}_{\mathcal{K}^{(2)}}^{*}\right)
$$


We evaluate the difference $\Delta_{j}\left(\boldsymbol{a}^{\prime}, \boldsymbol{a}^{*}\right)=u_{j}\left(\boldsymbol{a}_{\mathcal{K}^{(1)}}^{*}, \boldsymbol{a}_{\mathcal{K}^{(2)}}^{*}\right)-$ $u_{j}\left(\boldsymbol{a}_{\mathcal{K}^{(1)}}^{\prime}, \boldsymbol{a}_{\mathcal{K}^{(2)}}^{*}\right)$. We have

$$
\begin{aligned}
\Delta_{j}\left(\boldsymbol{a}^{\prime}, \boldsymbol{a}^{*}\right)= & \sum_{i \in \mathcal{K}^{(1)}} w_{j i} \mathbb{1}_{\left\{a_{j}^{*} \neq a_{i}^{*}\right\}}+\sum_{\ell \in \mathcal{K}^{(2)}} w_{j \ell} \mathbb{1}_{\left\{a_{j}^{*} \neq a_{\ell}^{*}\right\}} \\
& -\sum_{i \in \mathcal{K}^{(1)}} w_{j i} \mathbb{1}_{\left\{a_{j}^{*} \neq a_{i}^{\prime}\right\}}-\sum_{\ell \in \mathcal{K}^{(2)}} w_{j \ell} \mathbb{1}_{\left\{a_{j}^{*} \neq a_{\ell}^{*}\right\}} \\
= & \sum_{i \in \mathcal{K}^{(1)}} w_{j i}\left(\mathbb{1}_{\left\{a_{j}^{*} \neq a_{i}^{*}\right\}}-\mathbb{1}_{\left\{a_{j}^{*} \neq a_{i}^{\prime}\right\}}\right) .
\end{aligned}
$$

Now, under the assumptions that $\mathcal{K}^{(1)} \neq \emptyset$ and $w_{i j} \neq w_{k \ell}$ for all $(i, j, k, \ell) \in \mathcal{K}^{4}$, with $(i, j) \neq(k, \ell)$, it follows that

$$
\Delta_{j}\left(\boldsymbol{a}^{\prime}, \boldsymbol{a}^{*}\right) \neq 0,
$$

which contradicts the assumption in (9) and proves that such a partition $\mathcal{K}^{(1)}$ and $\mathcal{K}^{(2)}$ cannot exist. This completes the proof.

\section{APPENDIX B}

TRIAL AND ERROR - TYPE I [9] (TE-I)

In TE-I learning, for all $k \in \mathcal{K}$, player $k$ implements a state machine. At time interval $n$, a state is defined by the triplet:

$$
Z_{k}(n)=\left\{m_{k}(n), \bar{a}_{k}(n), \bar{u}_{k}(n)\right\},
$$

where $m_{k}(n)$ represents the "mood" of player $k$, that is, the way it reacts to the observation of its achieved utility; and $\bar{a}_{k} \in \mathcal{A}$ and $\bar{u}_{k} \in[0,1]$ represent a benchmark action and benchmark utility, respectively. There are four possible moods: content $(C)$, watchful $(C-)$, hopeful $(C+)$, discontent $(D)$, and thus, $m_{k}(n) \in\{C, C+, C-, D\}$. We use the notation $X \Leftarrow Y$ to indicate that variable $X$ takes the value of variable $Y$.

If at stage $n$ player $k$ is content, it uses the benchmarked action $\bar{a}_{k}(n)$ with probability $(1-\epsilon)$ and experiments a new action $a_{k}^{\prime}(n)$, with probability $\epsilon$. The utility achieved with action $a_{k}^{\prime}(n)$ is observed in the next time interval and we denote it by $u_{k}^{\prime}(n+1)$. If a player experiments and $u_{k}^{\prime}(n+1)<\bar{u}_{k}(n)$ then $Z_{k}(n+1) \Leftarrow Z_{k}(n)$, otherwise if $u_{k}^{\prime}(n+1)>\bar{u}_{k}(n)$, then, with probability $\epsilon^{G\left(u_{k}^{\prime}(n+1)-\bar{u}_{k}(n)\right)}$, it modifies its own benchmarks as follows: $\bar{u}_{k}(n+1) \Leftarrow$ $u_{k}^{\prime}(n+1)$ and $\bar{a}_{k}(n+1) \Leftarrow a_{k}^{\prime}(n)$. Here, $G(\cdot)$ is a strictly decreasing function that satisfies $0<G(\cdot)<\frac{1}{2}$.

If at stage $n$ player $k$ observes an increment or decrement in its utility without having experimented at the previous stage, then the mood becomes hopeful or watchful, according to the following rule: $(i)$ if $u_{k}^{\prime}(n+1)>\bar{u}_{k}(n)$ then, $m_{k}(n+1) \Leftarrow$ $C+, \bar{a}_{k}(n+1) \Leftarrow \bar{a}_{k}(n)$ and $\bar{u}_{k}(n+1) \Leftarrow \bar{u}_{k}(n)$; or $(i i)$ if $u_{k}^{\prime}(n+1)<\bar{u}_{k}(n)$, then $m_{k}(n+1) \Leftarrow C-, \bar{a}_{k}(n+1) \Leftarrow \bar{a}_{k}(n)$ and $\bar{u}_{k}(n+1) \Leftarrow \bar{u}_{k}(n)$. If player $k$ observes an improvement also at the next stage (i.e., $u_{k}^{\prime}(n+2)>\bar{u}_{k}(n+1)$ ), then the mood switches to content and the benchmark utility is updated with the new one: $m_{k}(n+2) \Leftarrow C$ and $\bar{u}_{k}(n+2) \Leftarrow u_{k}^{\prime}(n+1)$. On the contrary, if $u_{k}^{\prime}(n+2)<\bar{u}_{k}(n+1)$, then the mood switches to discontent: $m_{k}(n+2) \Leftarrow D$.

If player $k$ is discontent, it always takes a new action $a_{k}^{\prime}(n)$. When the corresponding utility $u_{k}^{\prime}(n+1)$ is observed, with probability $\epsilon^{F\left(u_{k}^{\prime}(n+1)\right.}$, it sets up the following parameters: $m_{k}(n+1) \Leftarrow C, \bar{u}_{k}(n+1) \Leftarrow u_{k}^{\prime}(n+1)$ and $\bar{a}_{k}(n+1) \Leftarrow$ $a_{k}^{\prime}(n+1)$. With probability $\left(1-\epsilon^{F\left(u_{k}^{\prime}(n+1)\right.}\right)$ it continues experimenting. The function $F$ is a strictly decreasing function that satisfies, $0<F(u)<\frac{1}{2 K}$.

\section{APPENDIX C \\ TRIAL AND ERROR - TYPE II [11] (TE-II)}

In TE-II learning, for all $k \in \mathcal{K}$, player $k$ implements a state machine similar to the case of TE-I. However, in this case, players only have two moods: content (C) and discontent (D). We describe the state dynamics as follows.

If at stage $n$ player $k$ is content, it chooses action $a_{k}^{\prime}(n)$ following the probability distribution

$$
\pi_{k, a_{k}}=\left\{\begin{array}{lll}
\frac{\epsilon^{c}}{\left|\mathcal{A}_{k}\right|-1} & \text { if } & \bar{a}_{k} \neq a_{k} \\
1-\epsilon^{c} & \text { if } & \bar{a}_{k}=a_{k} .
\end{array}\right.
$$

where, $\pi_{k, a_{k}}=\operatorname{Pr}\left(a_{k}^{\prime}(n)=a_{k}\right)$. If player $k$ uses its benchmarked action, i.e, $a_{k}^{\prime}(n)=\bar{a}_{k}$ and $u_{k}^{\prime}(n+1)=\bar{u}_{k}(n)$ then $Z_{k}(n+1) \Leftarrow Z_{k}(n)$. Otherwise, it adopts a new benchmarked action and utility: $\bar{u}_{k}(n+1) \Leftarrow u_{k}^{\prime}(n+1)$ and $\bar{a}_{k}(n+1) \Leftarrow a_{k}^{\prime}(n)$. With probability $\epsilon^{1-u_{k}^{\prime}(n+1)}$ it sets up $m_{k}(n+1) \Leftarrow C$ and with probability $1-\epsilon^{1-u_{k}^{\prime}(n+1)}$, it sets up $m_{k}(n+1) \Leftarrow D$.

If player $k$ is discontent, then it chooses its action $a_{k}^{\prime}(n)$ with uniform probability among all its possible choices. Moreover, independently of the benchmarked action $\bar{a}_{k}(n)$ and utility $\bar{u}_{k}(n)$, it updates them as follows: $\bar{u}_{k}(n+1) \Leftarrow u_{k}^{\prime}(n+1)$ and $\bar{a}_{k}(n+1) \Leftarrow a_{k}^{\prime}(n)$. With probability $\epsilon^{1-u_{k}^{\prime}(n+1)}$ it sets up $m_{k}(n+1) \Leftarrow C$ and with probability $1-\epsilon^{1-u_{k}^{\prime}(n+1)}$, it sets up $m_{k}(n+1) \Leftarrow D$. 